\title{
A Corollary of the Conant-Ashby Theorem Applied to Abio- genesis
}

\author{
Eugenio Darbesio \\ DOI: 10.33014/issn.2640-5652.3.2.darbesio.1
}

\section{Abstract}

From the Conant-Ashby theorem about the "good regulator" is possible to derive a corollary about the origin of life (OOL). This corollary introduces the concept of "good constructor." Then it is shown as nature, seen as a material system ruled by the laws of physics, cannot be a "good constructor" of the basic machinery necessary for a living cell. As a consequence OOL needs intelligent design.

Norbert Wiener defined cybernetics as "the science that deals with control and communication in systems and organisms"(Wiener, 1948). Biochemist Michael Behe states: "the essence of cellular life is regulation: the cell controls how much and what kinds of chemicals it makes; when it loses control, it dies" (Behe, 2006).

Given these statements on the importance of control, one wonders if cybernetics can say something about naturalistic origin of life (abiogenesis) and evolution. The answer is a bold yes. Even one of the theorems of cybernetics can provide us the basis for a disproof of abiogenesis. In theoretical cybernetics a fundamental result is the "good regulator theorem" by Roger C.Conant and William Ross Ashby (Conant and Ashby, 1970), henceforth referred to as the "C\&A theorem":

Theorem: The simplest optimal regulator $\mathrm{R}$ of a reguland $\mathrm{S}$ produces events $\mathrm{R}$ which are related to the events $\mathrm{S}$ by a mapping $\mathrm{h}: \mathrm{S} \rightarrow \mathrm{R}$.

Restated somewhat less rigorously, the theorem says that the best regulator of a system is one which is a model of that system in the sense that the regulator's actions are merely the system's actions as seen through a mapping $h$. The type of isomorphism here is that expressed (in the form used above) by

$$
\exists h: \forall i: \rho(i)=\mathrm{h}[\sigma(i)]
$$

where $\rho$ and $\sigma$ are the mappings that R and $\mathrm{S}$ impose on their common input I. [...] The effect of this theorem is to change the status of modelmaking from optional to compulsory.
In a sense the $C \& A$ theorem is intuitive: to regulate a system you must know the system. More the knowledge more good the regulation and control. Without knowledge it is likely you damage the system instead of regulating it. This is the reason why children are not allowed to drive cars and manage weapons, entrance in the aircraft cockpit is forbidden to passengers, etc..

Cybernetician Daniel L.Scholten, in his revisitation of the C\&A theorem (Scholten, 2010), stress its importance in many fields: "The C\&A theorem is a fundamental theorem of problem solving and as such shows us how to make meaningful progress toward the solution of any problem whatsoever." He adds:

As an example of a static control-model, consider an inexperienced cook attempting to make a roast duck with the help of a recipe. In this case, the system to be regulated consists of the various ingredients and kitchen tools to be used to create the meal, the dynamic good-regulator model is the human being doing the cooking, and the recipe is what we are calling the static "control-model".

The intuitive cook's example can be generalized to any constructor/assembler of systems. In this more general case, by paraphrasing Scholten, we can say that the system to be regulated consists of the various elements/parts and assembly tools to be used for the job, the "dynamic good-regulator model" is the "good constructor" making the construction, and the "design" is the static "control-model."

Also the constructor corollary of the C\&A theorem considered here is intuitive: to construct a system one must have it in his mind in any detail. In other words this corollary is consistent with any intelligent design scenario where a designer conceives before and eventually construct after a system. The designer must contain in his mind an internal isomorphism of the external design. This is the reason why laymen are not in charge of projecting nuclear plants, why to build houses they hire architects and so on. John von Neumann's in his research about automata perfectly expressed the "constructor corollary" this way: "Generally speaking, an automaton $A$, which can make an automaton $B$, must contain a complete description of $B$ 
and also rules on how to behave while effecting the synthesis" (von Neumann, 1966). The constructor corollary is coherent with causation theory: any cause virtually contains all its effects just from the beginning. It is consistent also with the potentiality principle: any system can produce only what is potentially contained in itself and its potentiality.

Here, as a follow-up or corollary of the C\&A theorem, I will consider its application to the question whether origin of life may have a naturalistic cause or an intelligent one. In other terms, can the natural forces alone, acting on sparse atoms and molecules, regulate and construct a first biological cell? To the goal it is necessary to construct the "GRC" (Genome + Ribosome + genomic Code), a dynamic chemical system that produces proteins from amino acids according to the genomic language and instructions. The GRC, a fundamental system in the molecular machinery of any biological cell, involves Turing machines (TM). In informatics a TM is defined as a finitestate machine able to write and read symbolic instructions in a memory device.

As part of the GRC, the DNA-polymerase molecular machine produces RNA from DNA (genome). The DNA, a couple of complementary strands of polimers composed of four symbols $\{A, T, G, C\}$ which can be written and read according to the "genetic code," may be thought of as a tape of a TM. Another part of the GRC is the ribosome. The ribosome is a molecular machine which translates messenger RNA (mRNA) and builds polypeptide chains (proteins) using amino acids carried by transfer RNA (tRNA).

Leonard Adleman, a mathematician who pioneered the socalled "DNA computing," in his first groundbreaking work, recognised that "biology and informatics, life and computers are tied toghether," and said that "it's hard to imagine something more similar to a Turing machine than the DNApolymerase" (Adleman, 1994). The DNA-polymerase is an important enzyme of the cell that is able, starting from a DNA strand, to produce another complementary DNA strand (complementarism means that $C$ changes to $G$ and $T$ changes to A). This nano-machine slides along the filament of the original DNA reading its bases and at the same time writes the complementary filament. As a TM begins an elaboration from a starting instruction on the tape likewise the DNA-polymerase needs a start mark telling it where to begin producing the complementary copy. Normally this mark consists of a DNA segment called a "primer." Analogously in the genetic code a stop codon (or termination codon) is a nucleotide triplet within messenger RNA that signals the termination of translation.

Biological computers are even more advanced, efficient and miniaturized than artificial ones. First the DNA language and the genetic code are highly optimized. Moreover the memory is used more efficiently. In fact, according to many researchers, often the same sequence of DNA contains multiple codes (e.g. it codifies for proteins and in the same time stores data related to other cellular processes or structures). Biological technology is superior because in multiple-coding DNA sometimes we derive different levels of interpretation from the same piece of code. Such astounding compression of data is very difficult to design, in fact it has rarely been attempted in human technology. It is clear that the problem of writing/reading memory in these cases of multiple interpretations becomes ever more unreachable by chance and necessity.

Back to the issue, can the system composed of the natural laws and randomness acting on atoms (henceforth "chance and necessity," "C\&N") regulate them until arriving to spontaneously assembly a GRC system? Since the isomorphism that is considered in the C\&A theorem must exist between GRC and $C \& N$ (if the latter must be the regulator of the construction process of the former) then we must analyze their structures and compare them (isomorphism is similarity of structure).

By doing that we discover that at least two things belonging to the structure of the GRC have no counterpart in C\&N (obviously this doesn't exclude other differences).

(1) First to be missing is the genetic code the GRC uses, i.e. the mapping between all the symbolic RNA nucleotide triplets XXX (where $X$ is $G$ or $A$ or $C$ or $U$ ) and the amino-acids. Example of the mapping: the symbol "CGU" (the molecular sequence cytosine / guanine / uracil) references arginine amino acid. This mapping is fully contingent and arbitrary. It is a free choice, as is free the choice of naming my cat Felix instead of Red. Nowhere the laws of physics and chemistry, neither explicitly nor implicitly, specify the arginine symbolic reference or other references of the genetic code. For $C \& N$ and its potentiality the genetic code simply doesn't exist.

What we argue here is similarly expressed by Albert Voie:

Due to the abstract character of function and sign systems, life is not a subsystem of natural laws...Life express both function and sign systems, which indicates that it is not a subsystem of the universe, since chance and necessity cannot explain sign systems, meaning, purpose and goals. (Voie, 2006)

Some could object that the interaction of randomness and physical laws causes things to happen that are not specified by any physical law (for example, the weird circuitous route that natural rivers generate, or crazy stone bridges from natural erosion). This object doesn't refute point (1) because circuitous route of rivers or stone bridges are not codes, that is abstract formalisms that map together abstract entities, whose reality transcends the physical plane. Routes of a river or stone bridges don't transcend the physical plane. 
(2) The GRC, being an instruction driven system, implements decision/control structures. The simplest of these programming structures has the form:

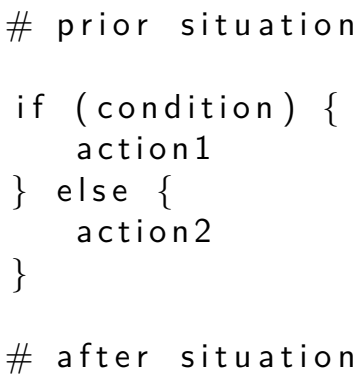

Note that a decision/control implies choice among two or more alternatives, depending on some conditions. A decision breaks the causal chain and inserts a choice discontinuity between "prior situation" and "after situation." This decision/choice is fully contingent and arbitrary. This kind of decision constructs can be nested ad libitum in a program to create complex control chains. Software, whatever be the material medium of the symbolic processing, is control. But complex control/regulation chains is something you find also countless times in the literature on cellular biology and systems biology.

Natural laws can be described by means of a basic set of equations. These equations represent the direct relations between variables, and directly assign values to these variables. Here a key point is the term "direct" and "directly." Example, in mechanics the Newtonian formula $\mathbf{F}=\mathbf{m}^{*} \mathbf{a}$ assigns a value to " $\mathbf{F}$ " when the values of the other two variables " $\boldsymbol{m}$ " and "a" are known. That is simple and unconditioned. The formula neither contains nor depends on any control structure related to some contingent/arbitrary constraint, which could insert a discontinuity in the Newton law. In other words, given any " $\mathbf{m}$ " and "a" there is no something like the following working on:

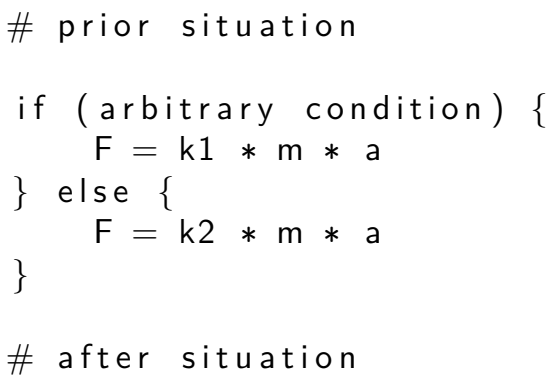

In the original formula $\mathbf{F}=\mathbf{m} * \mathbf{a}$, between any "prior situation" and "after situation," there is no discontinuity due to decisions that break the physicodynamic determinism by introducing free choices (above symbolized by the different coefficients $\mathbf{k}_{\mathbf{1}}$ and $\mathbf{k}_{2}$ ). This is an important point: in natural laws there aren't arbitrary contingent decisions like those that exist in software.
Natural laws have no choices, no freedom, they have only constraints. This is true for all physical laws, also when they are expressed as differential equations (e.g., wave equation, Maxwell's equations, Schrödinger equation, etc.).

Obviously if natural laws (necessity) don't have decision structures involving contingency and arbitrariness, for greater reason randomness (chance) has no decision power or freedom. In fact, randomness does not even have the minimal structure that natural laws have. Random events are fully uncorrelated, as by the definition of chance.

A critic could claim that interaction of physical objects can also create conditional discontinuities (for example, iron is normally not magnetic, but when a magnet is placed near a piece of iron it becomes magnetic). Again this criticism doesn't apply to our point (2) because in the statement "IF a magnet is placed near a piece of iron it becomes magnetic" the IF is static and part of nature (no choice, a piece of iron near a magnet becomes always magnetic). Differently, in the instruction driven systems considered in bio-cybernetic (or computer programming) the IFs are artificial, dependent on the highly specific configuration of the codes. Additionally, for control theory and $C \& A$, these statements are dependent on the holistic state of the system. For a biological example, in the cell a tRNA loaded with an amino acid doesn't always produce a piece of protein. It does it only IF the tRNA gets in the ribosome assembly machine. But this is a contingent choice, in the spacetime, of the cellular design. It is both artificial (dependent on code sequence) and holistic (dependent on the holistic state of the organism). In short, the "conditional discontinuity" of the iron-magnet is not a real conditional in the programming sense. Programming, in computers and biocybernetics, is freedom. Iron and magnets have no freedom.

To sum up: for the above two differences, the necessary isomorphism condition of the C\&A theorem is not satisfied. C\&N cannot be the "good constructor" of a GRC. It is necessary a constructor implyies the isomorphism between itself and the $\mathrm{GRC}$, i.e. an intelligent constructor.

In a sense the C\&A theorem is intuitive: to regulate a system one must know the system. Also the corollary considered here is intuitive: to construct a system one must have it in his mind. In short, if to regulate something one must know it, then even more one must know it to construct it ex novo.

Of course the C\&A theorem and its corollary perfectly agree with all the ideas of design theorists:

- William Dembski's "conservation of information" principle (Dembski, 1998)

- David Abel's "Formalism > Physicality $(F>P)$ " principle (Abel, 2011) 
- Donald Johnson: "while life uses the laws of chemistry and physics, those laws cannot define or explain life any more than the rules of grammar that were used during the preparation of a book define its content" (Johnson, 2010)

We have seen that $C \& N$ is unable to regulate the construction of the first GRC. The C\&A theorem prescribes an isomorphism between $C \& N$ and what it pretends to construct. Since $C \& N$ lacks an isomorphism with GRC, then no $C \& N$ process can produce a GRC. Since GRC is a key feature of any biological cell, then the cell cannot be produced by $C \& N$. Life didn't arise from a primordial soup this way. This means that an intelligent cause was at work. Because only intelligence is able to contain and inject models and formalisms into the systems it designs.

\section{References}

Abel, David (2011). The First Gene: The Birth of Programming, Messaging, and Formal Control. Longview Press.

Adleman, Leonard (1994). "Molecular Computation of Solutions to Combinatorial Problems". In: Science 266.5187, pp. 1021-1024. DOI: 10.1126/science.7973651.

Behe, Michael (2006). Darwin's Black Box.

Conant, R C and R Ashby (1970). "Every Good Regulator of a System Must be a Model of that System". In: International Journal of Systems Science 1.2, pp. 89-97.

Dembski, W A (1998). The Design Inference. Cambridge University Press.

Johnson, Donald (2010). Programming of Life.

Scholten, D L (2010). Every Good Key Must be a Model of the Lock it Opens (The Conant \& Ashby Theorem Revisited).

Voie, Albert (2006). "Biological Function and the Genetic Code are Interdependent". In: Chaos, Solitons, and Fractals 28.4.

von Neumann, J (1966). Theory of Self-Reproducing Automata. Urbana: University of Illinois Press.

Wiener, N (1948). Cybernetics: Or Control and Communication in the Animal and the Machine. MIT Press. 